\title{
MONTMORT'S PROBLEM, BURNSIDE'S LEMMA AND BELL'S NUMBERS
}

\author{
R. AVENHAUS \\ Universität der Bundeswehr München \\ Werner-Heisenberg-Weg 39, D-85577 Neubiberg \\ E-mail: avenhaus@informatik.unibw-muenchen.de
}

Received June 6, 2005; revised August 20, 2005

\begin{abstract}
For a set of $n$ discernible objects, the probability of choosing at random a permutation with $\mathrm{m}$ objects fixed is determined with the help of the generating function method. It is shown that the expected number of objects left fixed is one, which represents a special interpretation of Burnside's Lemma. Furthermore, the higher moments about the origin are represented by Stirling's numbers of the second kind, or simpler, by Bell's numbers, and the factorial moments are all one. A short discussion of an estimation problem concludes the paper.
\end{abstract}

Key words: Bell's numbers, Burnside's lemma, matching problem, Stirling's numbers

\section{Problem Formulation}

Let us consider $n$ discernible objects and all its $n$ ! permutations, and let us draw at random one of these permutations. Then the question arises as to the probability $p_{n, m}$ or of a given number of $m$ objects left fixed for any $m$ with $m=0,1, \ldots, n, n=1,2, \ldots$.

According to Feller [9] a special case of this matching problem or probleme des rencontres was formulated for the first time by Montmort in 1708 [6]. There are many, more or less serious illustrations of it. Rohatgi [12] gives as example the careless secretary who mixes letters and envelopes and hopes that at least a few letters get into the suitable envelopes. In Munich the story goes as follows: All inhabitants and guests attend the Oktoberfest and get drunk thus, the Lordmajor wants to know what the probability is that at least one or any given number of inhabitants and guests find their own beds.

In the preface of his book on combinatorics, Jacobs [10] points out that there are basically two different ways of studying combinatorics: Either one 
can follow the trend of the Rota school to systematize combinatorial ideas and proofs; as an example, take Aigner [2]. Or one can try, in the Indian-IsraelianHungarian style, to pose and to solve single problems in the spirit of some guiding ideas. Obviously, this paper is an example for the second approach.

In the following we will see that, in analyzing our problem, we will encounter some well known relations and names from the past. Some of the results, e.g., (2.11), (3.7) and (5.2), and also the interpretation of (3.1) are new, or more precisely, could not be found in the literature despite of intense investigations.

\section{Probability Distribution}

In order to tackle the problem, let us define $L_{n, m}, m=0, \ldots, n, n=1,2, \ldots$ as the number of possibilities to permutate $n$ objects such that precisely $m$ objects remain fixed, and let us define $L_{0,0}=1$. The following self-explaining relation,

$$
L_{n, m}=\left(\begin{array}{c}
n \\
m
\end{array}\right) L_{n-m, 0},
$$

is crucial for all subsequent considerations. Furthermore, let us define $L_{n}$ as the number of possibilities to permutate $n$ objects such that at least one object remains fixed and let us put $L_{0}=0$. With $(2.1)$, we obtain

$$
L_{n}=\sum_{i=1}^{n} L_{n, i}=\sum_{i=1}^{n}\left(\begin{array}{c}
n \\
i
\end{array}\right) L_{n-i, 0} .
$$

Now we have

$$
L_{n, 0}+L_{n}=n !,
$$

therefore we get the following recursive relation for $\mathrm{L}_{n}$ :

$$
L_{n}=\sum_{i=1}^{n}\left(\begin{array}{c}
n \\
i
\end{array}\right) \cdot\left((n-i) !-L_{n-i}\right), \quad L_{0}=0 .
$$

Let furthermore, $p_{n}$ be defined as the probability to choose one permutation of $n$ objects such that at least one object is left fixed. Then we have by Laplace's rule

$$
p_{n}=\frac{L_{n}}{n !}
$$

and therefore, using (2.4), we get the following recursive relation for the $p_{n}$ :

$$
p_{n}=\sum_{i=1}^{n} \frac{1}{i !}\left(1-p_{n-i}\right), \quad p_{0}=0 .
$$

We solve this recursive relation by means of the generating function method. We define 


$$
f(x)=\sum_{n=1}^{\infty} p_{n} x^{n}
$$

(formally, without discussing convergence questions) and get with the help of the recursive relation $(2.5)$

$$
f(x)=\sum_{n=1}^{\infty} \sum_{i=1}^{n} \frac{1}{i !} x^{n}-\sum_{n=1}^{\infty} \sum_{i=1}^{n} \frac{p_{n-i}}{i !} x^{n} .
$$

Changing summations and transforming the summation variable $n$ to $k$ according to $n=i+k$ we obtain

$$
f(x)=\sum_{i=1}^{\infty} \frac{x^{i}}{i !} \sum_{k=0}^{\infty} x^{k}-\sum_{i=1}^{\infty} \frac{x^{i}}{i !} \sum_{k=0}^{\infty} p_{k} x^{k},
$$

or, performing the sums,

$$
f(x)=\left(e^{x}-1\right) \frac{1}{1-x}-\left(e^{x}-1\right)\left(f(x)+p_{0}\right) .
$$

With (2.6), we have $p_{0}=0$. Thus, we get the explicit form of the generating function as follows,

$$
f(x)=\frac{1-e^{-x}}{1-x} \text { for } 0 \leq x<1 .
$$

In order to get the probabilities $p_{n}$, we may use again the power series expansions of $1-e^{-x}$ and $\frac{1}{1-x}$ and multiply them. According to (2.6), $p_{n}$ is than found as the coefficient of $x^{n}$.

Alternatively, one can determine $p_{n}$ according to

$$
p_{n}=\left.\frac{1}{n !} f^{(n)}(x)\right|_{x=0}, \quad \text { for } n=1,2, \ldots
$$

Using Leibniz' formula for the $n$-th derivative of the product $u v$,

$$
(u v)^{(n)}=\sum_{j=0}^{n}\left(\begin{array}{c}
n \\
i
\end{array}\right) u^{(i)} v^{(n-i)}
$$

and taking $u=1-e^{-x}$ and $v=\frac{1}{1-x}$ we get

$$
p_{n}=1-\sum_{i=0}^{n} \frac{(-1)^{i}}{i !} \quad \text { for } n=1,2, \ldots
$$

Special values are

$$
p_{0}=0, p_{1}=1, p_{2}=\frac{1}{2}, p_{3}=\frac{2}{3} \approx 0,666, p_{4}=\frac{5}{8} \approx 0,625 .
$$

For $n \rightarrow \infty$, we get 


$$
\lim _{n \rightarrow \infty} p_{n}=1-\sum_{i=0}^{n} \frac{(-1)^{i}}{i !}=1-\frac{1}{e} \approx 0.6321 .
$$

Of course, it can be shown directly that (2.9) satisfies (2.6). For that purpose one has to prove the identity

$$
\sum_{i=0}^{n} \frac{1}{i !} \sum_{j=0}^{n-i} \frac{(-1)^{j}}{j !}=1, \quad \text { for } n=1,2, \ldots
$$

which, according to Knuth [11], was already known to Stirling [14].

It should be mentioned here (see also Jacobs, [10]) that in 1736 L. Euler [8] has given the following recursive relation for the so-called Rencontre numbers $D_{n}:=n !-L_{n}$, i.e., the number of possibilities to permutate $n$ objects such that no object remains fixed,

$$
D_{n+1}=n\left(D_{n-1}+D_{n}\right), \quad D_{0}=1, \quad D_{1}=0,
$$

which can be understood as follows: we count, how many permutations of $n+1$ objects without fixed objects can be obtained. First, on place $n+1$ we put a number $k$ different from $n+1$, there are $n$ possibilities. The possibilities to occupy the remaining $n$ places without fixed objects, can be separated into two classes.

1. In the first class, place $k$ is occupied by object $n+1$, and there remain the $n-1$ places different from $k$ and $n+1$ and therefore, $D_{n-1}$ possibilities to occupy them without fixed object.

2. In the second class, there are all occupations of places $1, \ldots, n$ with objects $1, \ldots, k-1, k+1, \ldots, n+1$, where object $n+1$ for place $k$ and any object $j$ for place $j$ is forbidden, i.e. $D_{n}$ possibilities.

This recursion leads to the same formula for our $L_{n}$ (with different initial values, of course) and therefore, to the following recursive relation for $p_{n}$ :

$$
(n+1) p_{n+1}=n p_{n}+p_{n-1}, \quad p_{0}=0, \quad p_{1}=1 .
$$

Also, it should be mentioned, that we can get (2.9) as well, if we apply the exclusion inclusion principle to the event $\cup_{i=1}^{n} A_{i}$, where $A_{i}$ is the event that the $i$-th object remains fixed (see, e.g., Rohatgi, [12]). Whereas this way we obtain a better insight into the structure of (2.9), the way used here leads us immediately to the determination of the more general probabilities.

Let us define the probability $p_{n, m}$ that $m$ out of $n$ objects remain fixed ("matches"). It is given by

$$
\begin{array}{r}
p_{n, m}=\frac{L_{n, m}}{n !}=\frac{1}{n !}\left(\begin{array}{c}
n \\
m
\end{array}\right) L_{n-m, 0}=\frac{1}{n !}\left(\begin{array}{c}
n \\
m
\end{array}\right) \\
\left((n-m) !-L_{n-m}\right) \\
=\frac{1}{m !}\left(1-\frac{L_{n-m}}{(n-m) !}\right)
\end{array}
$$


or finally, with (2.5), by

$$
p_{n, m}=\frac{1}{m !}\left(1-p_{n-m}\right),
$$

where $p_{n}$ is given by (2.9). Explicitly, we get

$$
p_{n, m}=\frac{1}{m !} \sum_{i=0}^{n-m} \frac{(-1)^{i}}{i !}, \text { for } n=1,2, \ldots, m=0,1, \ldots n .
$$

Special and intuitively comprehensible cases are

$$
p_{n, 0}=\sum_{i=0}^{n} \frac{(-1)^{i}}{i !}=1-p_{n}, \quad p_{n, n-1}=0, \quad p_{n, n}=\frac{1}{n !} .
$$

Furthermore, we obviously have

$$
p_{n}=\sum_{m=1}^{n} p_{n, m}
$$

which also can be shown formally, if (2.11) is used since this way, again the recursive relation (2.6) is obtained. Also, if we use (2.12), we arrive again at the identity mentioned previously.

The normalization of the probabilities $p_{n, m}$ is obtained according to

$$
\sum_{m=0}^{n} p_{n, m}=\sum_{m=1}^{n} p_{n, m}+p_{n, 0}=p_{n}+1-p_{n}=1, \text { for } n=1,2, \ldots
$$

Finally, from (2.11) we get the useful recursive relation

$p_{n-1, m-1}=\frac{1}{(m-1) !}\left(1-p_{n-m}\right)=m p_{n, m}$, for $n=1,2, \ldots, m=1,2, \ldots, n$.

Returning to the $L_{n, m}$ as given by (2.1), this recursive relation leads immediately to

$$
n L_{n-1, m-1}=m L_{n, m} .
$$

This form we can obtain directly from (2.1),

$$
L_{n, m}=\left(\begin{array}{c}
n \\
m
\end{array}\right) L_{n-m, 0},
$$

by writing it down again for $n-1$ and $m-1$,

$$
L_{n-1, m-1}=\left(\begin{array}{c}
n-1 \\
m-1
\end{array}\right) L_{n-m, 0},
$$

and eliminating $L_{n-m, 0}$ from both forms. 


\section{Moments about the Origin}

In order to determine the expected number $\mu_{n}$ of objects left fixed, we use the recursive relation (2.15). We get for all $n=1,2, \ldots$

$$
\mu_{n}:=\sum_{m=1}^{n} m p_{n, m}=\sum_{m=1}^{n} p_{n-1, m-1}=\sum_{i=0}^{n-1} p_{n-1, i}=1,
$$

independent of $n$. If we use again (2.10), we get the relation

$$
\sum_{m=1}^{n} m L_{n, m}=n !
$$

which means that the total number of objects left fixed under all permutations is just the number of all permutations. This is a special case of Burnside's Lemma (see, e.g., Rotman, [13]) which, according to Burnside himself, was first proved by Frobenius in 1887. It should be mentioned that according to the author's knowledge, so far this proof, or vice versa, this interpretation of Burnside's Lemma, has not yet been documented in the literature.

Also, it should be mentioned here, that the expected value (3.1) can be determined without knowledge of the distribution (2.12) by using the linearity of the expectation (see Aigner, [3]).

In general, the $k$-th moment $\mu_{n}^{(k)}$ about the origin of the number of objects left fixed is defined as

$$
\mu_{n}^{(k)}=\sum_{m=0}^{n} m^{k} p_{n, m}, \text { for } k=1,2, \ldots n, \mu_{n}^{(0)}=1 .
$$

Using the explicit form (2.12) of the $p_{n, m}$ we get

$$
\mu_{n}^{(k)}=\sum_{m=0}^{n} \sum_{i=0}^{n-m} \frac{(-1)^{i} m^{k}}{m ! i !}
$$

which gives with $m \rightarrow l, i \rightarrow m=i+l$

$$
\mu_{n}^{(k)}=\sum_{l=0}^{n} \sum_{m=l}^{n} \frac{(-1)^{m-l} l^{k}}{l !(m-l) !}, \text { for } k=1, \ldots, n,
$$

or, after another change of the order of summations

$$
\mu_{n}^{(k)}=\sum_{m=0}^{n} \frac{1}{m !} \sum_{l=0}^{m}(-1)^{m-l}\left(\begin{array}{c}
m \\
l
\end{array}\right) l^{k}=\sum_{m=0}^{k} S_{k, m} \text { for } k=1,2, \ldots, n,
$$


since $S_{k, m}=0$ for $m>k$. Now $S_{k, m}$ is Stirling's number of the second kind [14], explicitly given by

$$
S_{k, m}=\frac{1}{m !} \sum_{l=0}^{m}(-1)^{m-l}\left(\begin{array}{c}
m \\
l
\end{array}\right) l^{k}, \text { for } m \leq k
$$

(see, e.g., Abramovitz and Stegun, [1] ).

Since Stirling's number $S_{k, m}$ is the number of ways of partitioning a set of $k$ elements into $m$ non-empty subsets, (3.4) says that the $k$-th moment about the origin of the number of objects left fixed under a randomly chosen permutation of $n$ objects equals the number of ways of partitioning a set of $k$ objects into non-empty subsets. Now, this number, which we call $B_{k}$, is just the $k$-th Bell number (after E.T. Bell, see Becker and Riordan, [4]) which can be calculated recursively according to

$$
B_{k+1}=\sum_{l=0}^{k}\left(\begin{array}{l}
k \\
l
\end{array}\right) B_{l}, \quad B_{0}:=l
$$

and which is, after Dobinski [7], explicitly given by

$$
B_{k}=\frac{1}{e} \sum_{l=0}^{\infty} \frac{l^{k}}{l !}, \text { for } k=1,2, \ldots, n .
$$

As a result, the $k$-th moment about the origin is given by the $k$-th Bell number

$$
\mu_{n}^{(k)}=B_{k}, \text { for } k=1,2, \ldots, n,
$$

it does not depend on $n$. In fact, from (3.3) we could have obtained directly by use of (2.15)

$$
\begin{aligned}
\mu_{n}^{(k+1)} & =\sum_{m=1}^{n} m^{k} p_{n-1, m-1}=\sum_{r=0}^{n-1}(r+1)^{k} p_{n-1, r} \\
& =\sum_{r=0}^{n-1} \sum_{l=0}^{k}\left(\begin{array}{l}
k \\
l
\end{array}\right) r^{l} p_{n-l, r}=\sum_{l=0}^{k}\left(\begin{array}{l}
k \\
l
\end{array}\right) \mu_{n-l}^{(l)}, \mu^{(0)}=1
\end{aligned}
$$

i.e., using the independence of the $\mu_{n}^{(k)}$ of $n$, the recursive relation (3.6) for Bell's numbers. Special values are

$$
B_{0}=1, \quad B_{1}=1, \quad B_{2}=2, \quad B_{3}=5, \quad B_{4}=15 .
$$




\section{Factorial Moments}

The $k$-th factorial moment is defined as

$$
\mu_{n}^{[k]}:=\sum_{m=l}^{n} m(m-1) \cdots(m-k+1) p_{n, m}
$$

with (2.15) we get

$$
\mu_{n}^{[k]}:=\sum_{m=2}^{n}(m-1) \cdots(m-k+1) p_{n-1, m-1}=\mu_{n-1}^{[k-1]},
$$

and so on. This means, because of $\mu_{n}^{[1]}=1$ for $n=1,2, \ldots$

$$
\mu_{n}^{[k]}=1, \text { for } k=1,2, \ldots, n=1,2, \ldots
$$

There is an alternative approach. The $k$-th factorial moment can be expressed by the moments about the origin (see also Berg, 1983 [5]).

$$
\mu^{[k]}=\sum_{j=0}^{k} s_{k, j} \mu^{(j)}
$$

or, using (3.4), by

$$
\mu^{[k]}=\sum_{j=0}^{k} s_{k, j} \sum_{i=0}^{j} S_{j, i}
$$

where $s_{k, j}$ and $S_{j, i}$ are Stirling's numbers of the first and second kind. This can be written as

$$
\mu^{[k]}=\sum_{i=0}^{k} \sum_{j=i}^{k} s_{k, j} S_{j, i}
$$

which leads immediately, by use of the orthogonality of both kinds of Stirling's numbers, back to (4.1). In passing, it should be mentioned that (4.3), (3.7) and (4.2) give an interesting relation between Bell's and Stirling's numbers of the first kind,

$$
\sum_{j=0}^{k} s_{k, j} B_{j}=1
$$

Inversely, the moments about the origin can be represented by the factorial moments with the help of Stirling's numbers of the second kind as

$$
\mu^{(k)}=\sum_{i=0}^{k} S_{k, i} \mu^{[k]}
$$

which, with (4.1), gives again (3.4), and which this way provides another explanation for the latter form. 


\section{Central Moments}

The $k$-th central moment $\tilde{\mu}_{n}^{(k)}$ of the number of objects left fixed is because of (3.1) is defined as

$$
\tilde{\mu}_{n}^{(k)}:=\sum_{m=0}^{n}(m-1)^{k} p_{n, m}
$$

special cases are

$$
\tilde{\mu}_{n}^{(0)}=1, \quad \tilde{\mu}_{n}^{(1)}=0, \quad \tilde{\mu}_{n}^{(2)}=\sigma_{n}^{2}=1 .
$$

For $k \geq 1$, we get from (4.3)

$$
\begin{aligned}
\tilde{\mu}_{n}^{(k)} & =(-1)^{k} p_{n, 0}+\sum_{m=2}^{n}(m-1)^{k} p_{n, m} \\
& =(-1)^{k} p_{n, 0}+\sum_{i=1}^{n-1} i^{k} p_{n, i+1} .
\end{aligned}
$$

Now, with (2.15), written as

$$
p_{n-1, j}=(i+1) p_{n, j+1},
$$

we get with (3.3)

$$
\begin{aligned}
\tilde{\mu}_{n}^{(k)} & =(-1)^{k} p_{n, 0}+\sum_{i=1}^{n-1} i^{k-1}(i+1-1) p_{n, i+1} \\
& =(-1)^{k} p_{n, 0}+\mu_{n-1}^{(k-1)}-\sum_{j=0}^{n}(j-l)^{k-1} p_{n, j}+(-1)^{k-1} p_{n, 0},
\end{aligned}
$$

or, finally

$$
\tilde{\mu}_{n}^{(k)}=\mu_{n-1}^{(k-1)}-\tilde{\mu}_{n}^{(k-1)} .
$$

The solution of this recursive relation is

$$
\tilde{\mu}_{n}^{(k)}=\sum_{l=0}^{k-1}(-1)^{l} \mu_{n-1}^{(k-1-l)}+(-1)^{k}
$$

or, if we define $\mu^{(-1)}:=B_{-1}:=1$ and use (3.7)

$$
\tilde{\mu}_{n}^{(k)}=\sum_{m=0}^{k}(-1)^{k-m} B_{m-1} \quad \text { for } k=0,1,2, \ldots, n=1,2, \ldots
$$

Even though this form look very concise, one must not forget that the moments about the origin, explicitly written, can only be represented by twofold finite sums, and therefore the central moments can be represented by threefold finite sums. 


\section{Note on Estimation}

We ask for an estimate of the total number $n$ of objects available if a sample of objects left fixed is given. For our Munich example this would mean that, with the help of the number of people who have found their own beds, the number of inhabitants and guests of Munich is estimated.

The maximum likelihood method does not work: taking the maximum of $p_{n, m}$ as given by (2.12) with respect to $n$ for fixed $m$ with $n \geq m$, one obtains the estimate $\hat{n}=m$ which is, because of (3.1), far from being an unbiased estimate of $n$.

In general, for a factorial series distribution, a special case of which we have discussed here, an unbiased estimate of $n$ is obtained as follows (see again Berg, [5]).

Let $\tilde{p}_{n, x}$ be the probability to choose one permutation of $n$ objects such that $x$ objects are not left fixed ("nonmatches"). With (2.12) and $x=n-m$ we obtain

$$
\tilde{p}_{n, x}=p_{n, n-x}=\frac{1}{(n-x) !} \sum_{i=0}^{x} \frac{(-1)^{i}}{i !}, \text { for } n=1,2, \ldots, x=0,1, \ldots, n,
$$

which we write as

$$
\tilde{p}_{n, x}=\frac{a_{x}}{(n-x) !} ; \quad a_{x}:=\sum_{i=0}^{x} \frac{(-1)^{i}}{i !} .
$$

Then, an unbiased estimate of $n$ is given by

$$
\hat{n}:=\frac{a_{x-1}}{a_{x}}+x
$$

since we have

$$
E(\hat{n})=\sum_{x=1}^{n}\left(\frac{a_{x-1}}{a_{x}}+x\right) \tilde{p}_{n, x}=\sum_{x=1}^{n}\left(\frac{a_{x-1}}{(n-x) !}+\sum_{x=1}^{n} x \tilde{p}_{n, x}\right.
$$

and therefore, because of (3.1),

$$
E(\hat{n})=\sum_{y=0}^{n-1} \frac{a_{y}}{(n-1-y) !}+n-1=\sum_{y=0}^{n-1} \tilde{p}_{n-1, y}+n-1=1+n-1=n
$$

It should be mentioned, however, that this estimate is not very satisfying. For large values of $n$ and consequently, for large values of $x$, one has approximately $\hat{n}=1+x$, which is reasonable because of $(3.1)$, but which means that practically all objects have to be counted. (In the case of Munich nearly all inhabitants and guests will have to report that they did not find their own beds).

In addition, $\hat{n}=x+1$ itself represents an unbiased estimate of $n$, and it is not known which of the two estimates has the smaller variance, since the 
variance of the estimate (6.2) is not known. In other words, it is not clear whether or not the estimate (6.2) for $n$ is at all worthwhile.

\section{References}

[1] M. Abramovitz and I. A. Stegun. Handbook of Mathematical Functions. Dover, New York. Last Edition

[2] M. Aigner. Combinatorial Theory. Springer, Berlin Heidelberg, 1979.

[3] M. Aigner. Diskrete Mathematik. Vieweg Braunschweig, 1993.

[4] H.-W. Becker and J. Riordan. The arithmetic of Bell and Stirling Numbers. American Journal of Mathematics, 70, 385 - 94, 1948.

[5] S. Berg. Factorial series distributions. In: Encyclopedia of Statistical Sciences, volume 3, Wiley, New York, 20, 1983.

[6] Pierre Rémond de Montmort (1678 1719). Essai d'analyse sur les jeux de Hasard. Paris, 1708.

[7] G. Dobinski. Summierung der Reihe $\sum n^{m} / n$ ! fiür $m=1,2,3,4,5, \ldots$, . Archiv für Mathematik und Physik, 61, 333 - 6, 1877.

[8] L. Euler. Solutio problematis ad Geometriam Situs pertinentis. Comm. Acad. Sci. Petr., 8, 128 - 140. Gesammelte Werke Band 7, 1 - 10, 1736

[9] W. Feller. An Introduction to Probability Theory and its Applications I. Wiley, New York, 3rd Edition, 1968.

[10] K. Jacobs. Einführung in die Kombinatorik. Walter de Gruyter, Berlin, 1983.

[11] D.E. Knuth. The Art of Computer Programming I. Addison-Wesley, 1975. 2nd Edition

[12] V. Rohatgi. An Introduction to Probability Theory and Mathematical Statistics. Wiley, 1976.

[13] J.J. Rotman. An Introduction to the Theory of Groups. Springer Berlin Heidelberg, 4th Edition, 1995.

[14] J. Stirling. Methodus Differentialis sive Tractatus de Summatione et Interpolatione Serierum Infinitarium. 1730. (English Translation: F. Holliday, The Differential Method, London 1749) 


\section{Montmorto uždavinys, Bernsaido lema ir Belo skaičiai}

R. Avenhaus

Darbe nagrinèjami klasikiniai kombinatorikos uždaviniai su tam tikra tikimybine interpretacija. Autorius taiko generuojančių funkcijų metodą įvairiems momentams skaičiuoti. Kai kurios iš įrodomų straipsnyje formulių nèra gerai žinomos kombinatorinejje analizejje. Kaip atskiri šių formulių rezultatai gaunami klasikiniai Stirlingo ir Belo skaičių sąryšiai. Straipsnyje pareikta trumpa nagrinèjamų uždavinių apžvalga. 